\title{
Predicting ambient aerosol thermal-optical reflectance (TOR) measurements from infrared spectra: extending the predictions to different years and different sites
}

\section{Reggente et al.}

Correspondence to: S. Takahama (satoshi.takahama@epfl.ch)

and A. M. Dillner (amdillner@ucdavis.edu)

The copyright of individual parts of the supplement might differ from the CC-BY 3.0 licence. 
S1. FT-IR OC

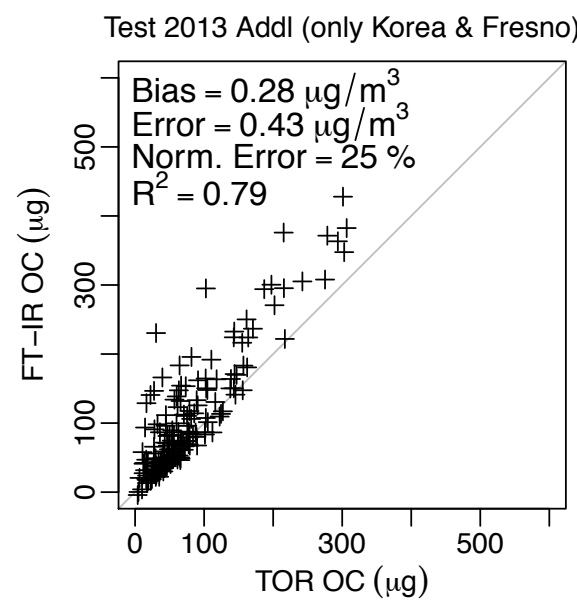

Figure S1. Scatterplot and performance metrics between FT-IR OC and TOR OC of the Korea and Fresno sites (Site ID 10 and 11 respectively) calibrated with the Calibration 2011 set. Concentration units of $\mu \mathrm{g} \mathrm{m}^{-3}$ for bias and error are based on the IMPROVE nominal volume of $32.8 \mu \mathrm{g} \mathrm{m}^{-3}$.

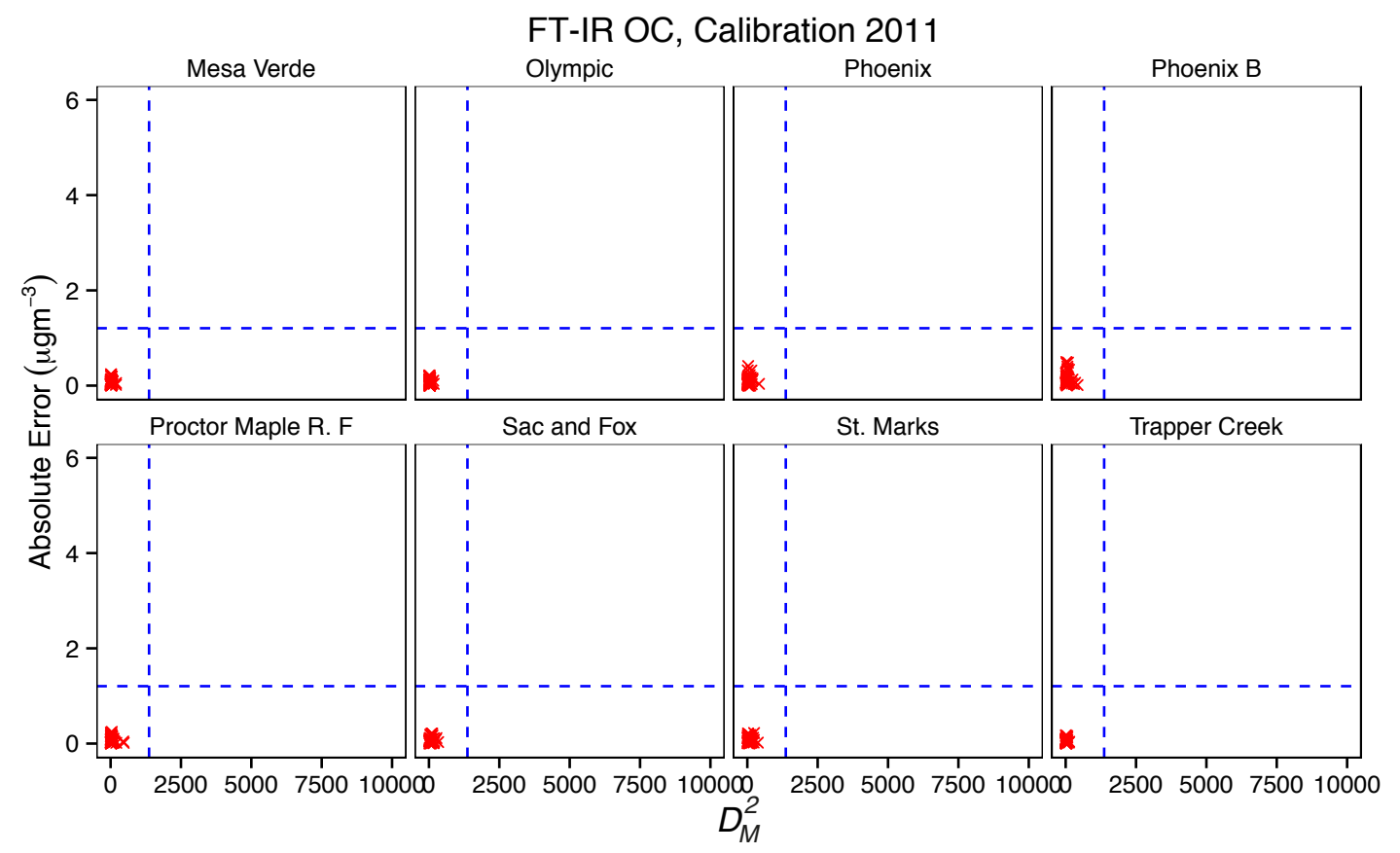

Figure S2. FT-IR OC, Calibration 2011. Anticipation of the prediction error. Mahalanobis distances per sample and site (between the scores of each dataset described in Section 2.1 and the centroid of the calibration dataset) against absolute errors (between TOR OC and FT-IR OC). Blue dashed lines represents the boundaries used for classification as explained in Section 2.4. 
FT-IR OC, Test 2011

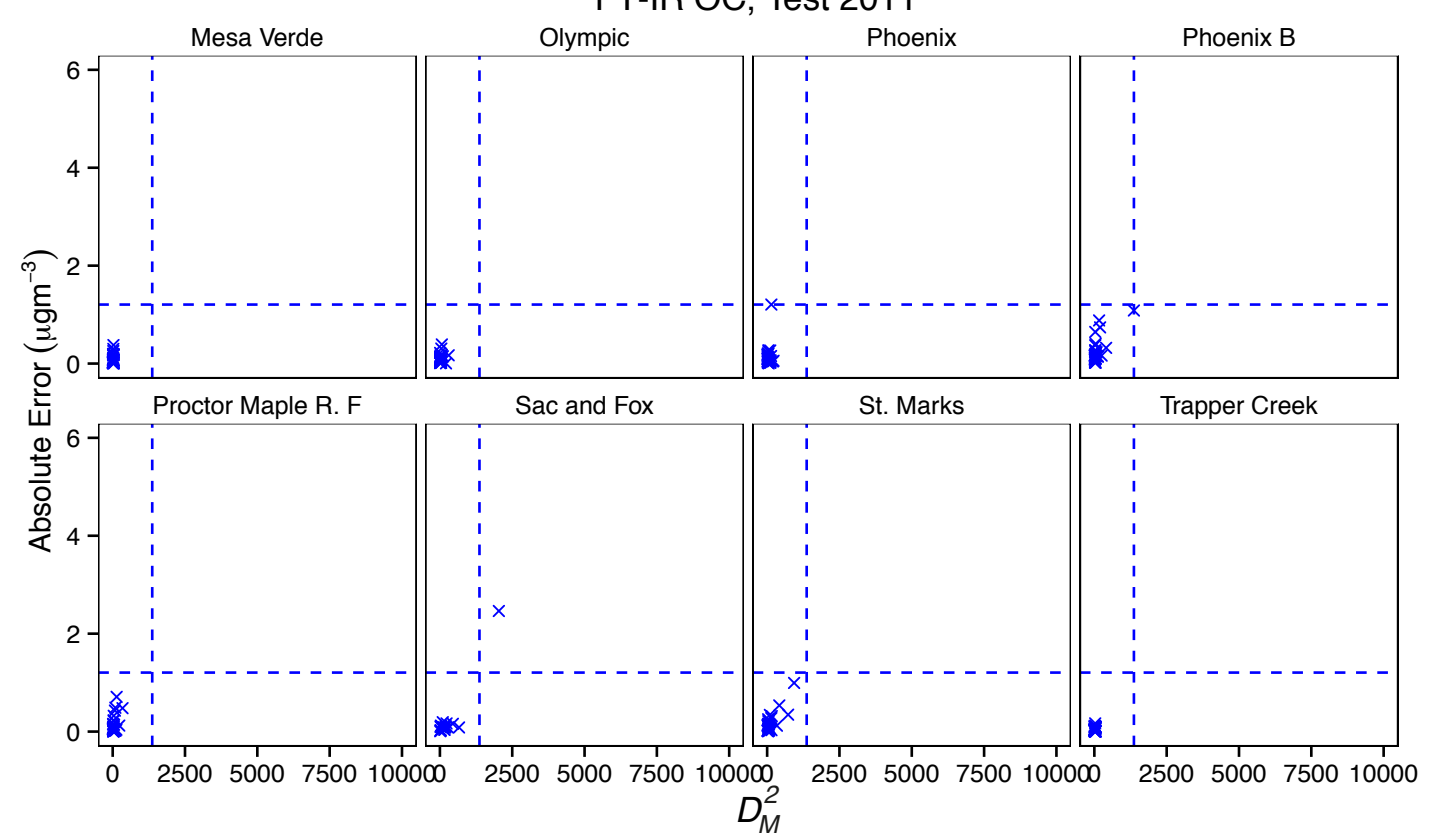

Figure S3. FT-IR OC, Test 2011. Anticipation of the prediction error. Mahalanobis distances per sample and site (between the scores of each dataset described in Section 2.1 and the centroid of the calibration dataset) against absolute errors (between TOR OC and FT-IR OC). Blue dashed lines represents the boundaries used for classification as explained in Section 2.4. The sample from Sac and Fox site that lies in the TP sector is the sample that we have considered as outlier with respect to the other sample in the same site, and therefore not used to calculate the values of the classification boundaries.

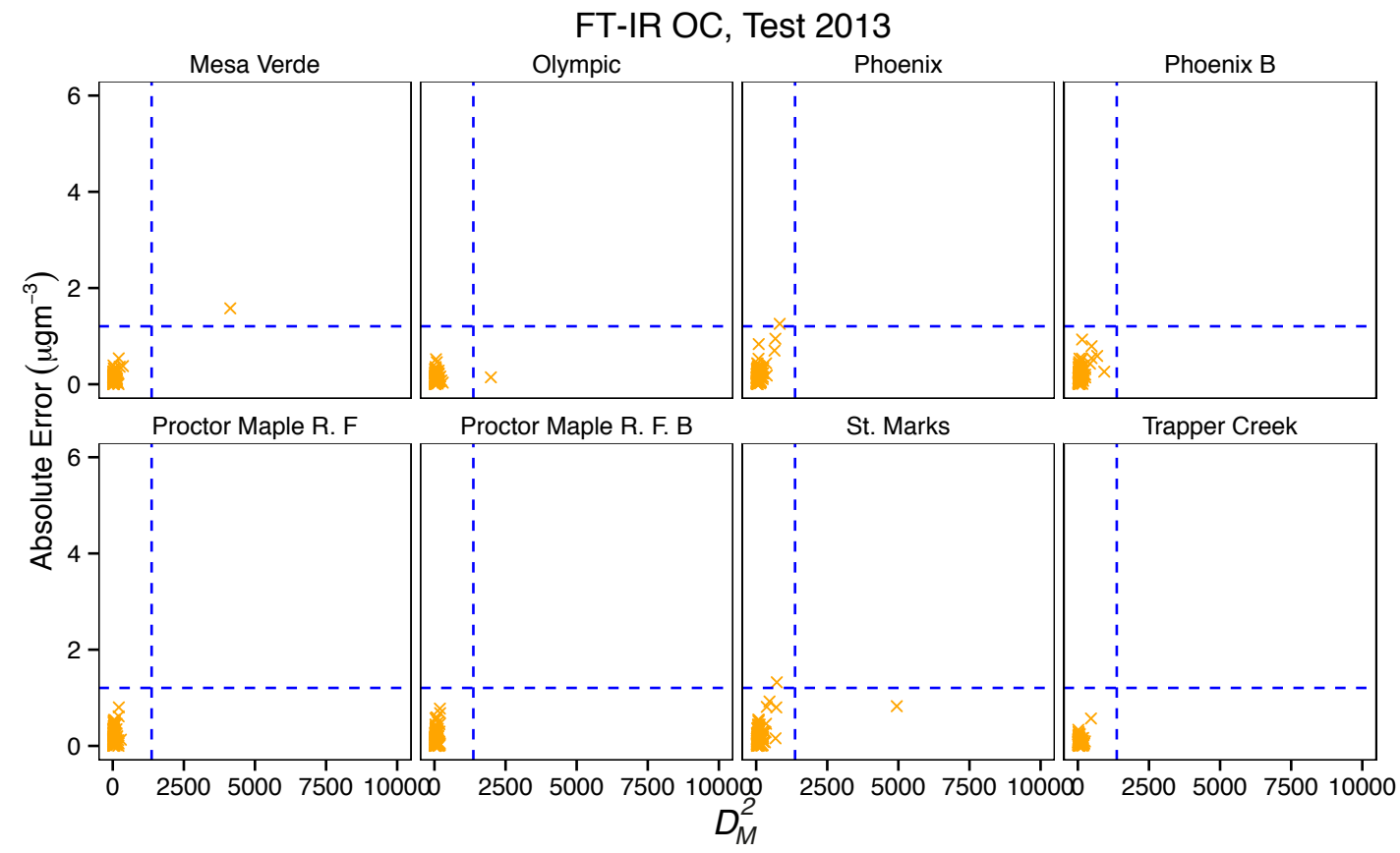

Figure S4. FT-IR OC, Test 2013. Anticipation of the prediction error. Mahalanobis distances per sample and site (between the scores of each dataset described in Section 2.1 and the centroid of the calibration dataset) 
against absolute errors (between TOR OC and FT-IR OC). Blue dashed lines represents the boundaries used for classification as explained in Section 2.4.

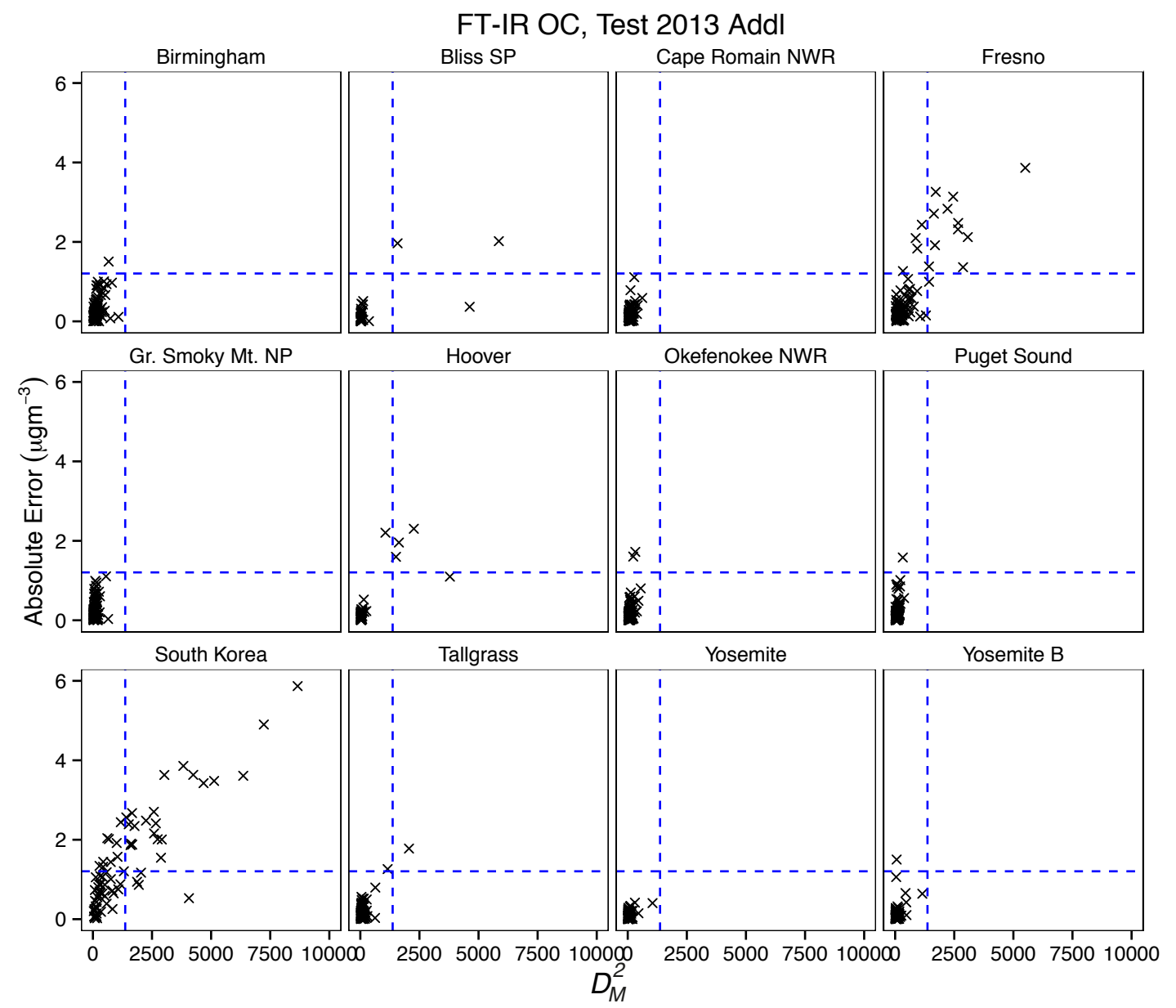

Figure S5. FT-IR OC, Test 2013 Addl. Anticipation of the prediction error. Mahalanobis distances per sample and site (between the scores of each dataset described in Section 2.1 and the centroid of the calibration dataset) against absolute errors (between TOR OC and FT-IR OC). Blue dashed lines represents the boundaries used for classification as explained in Section 2.4. 


\section{Calibration 2011}
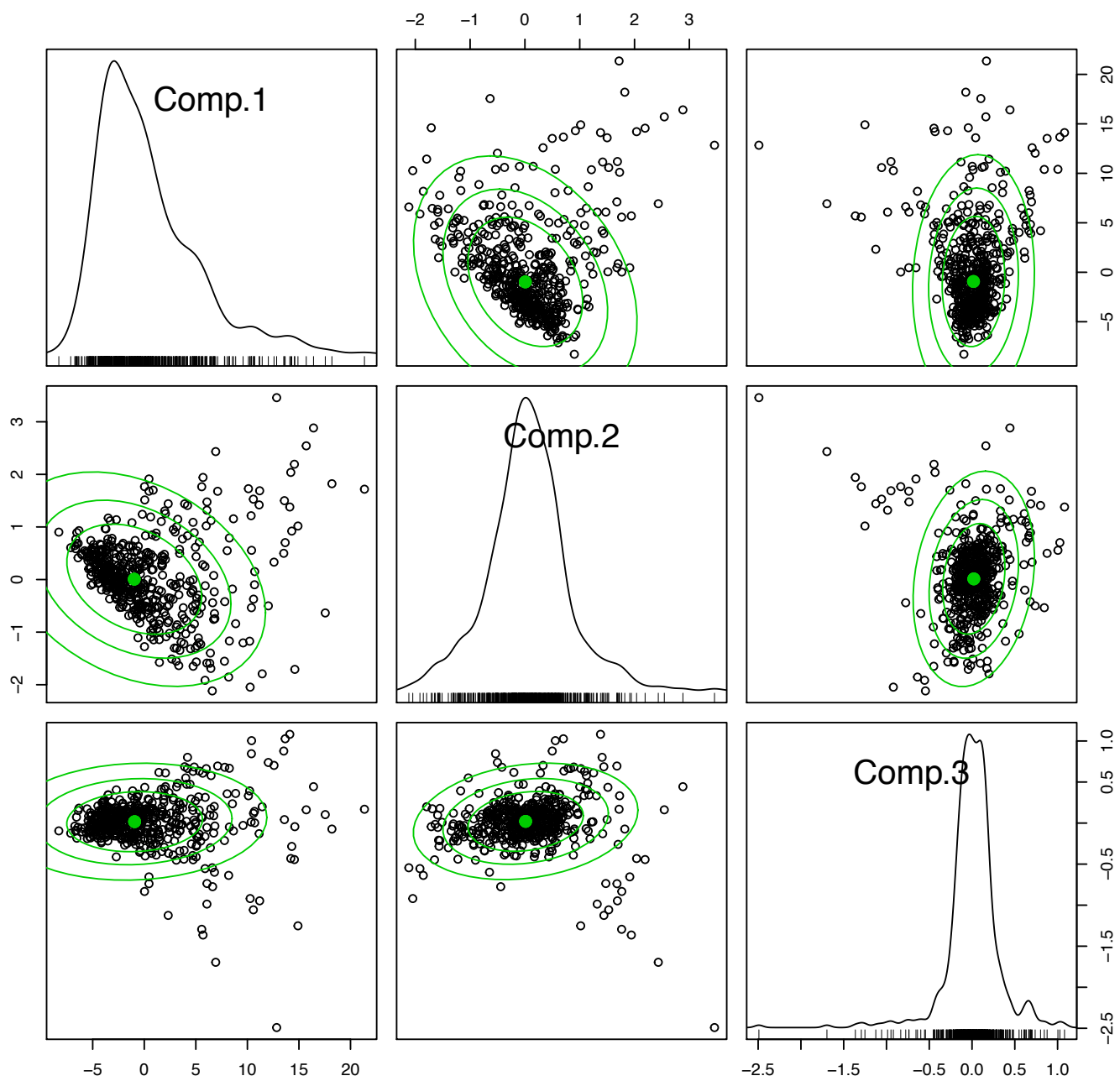

Figure S6. OC, Scatterplot matrix between the first three scores of the Calibration 2011 dataset. The green ellipses are the contours (one to three standard deviations) of the bivariate normal distribution between the scores' combinations. 


\section{S2. FT-IR EC}

Test 2013 Addl (only Fresno)

Test 2013 Addl (only Korea)

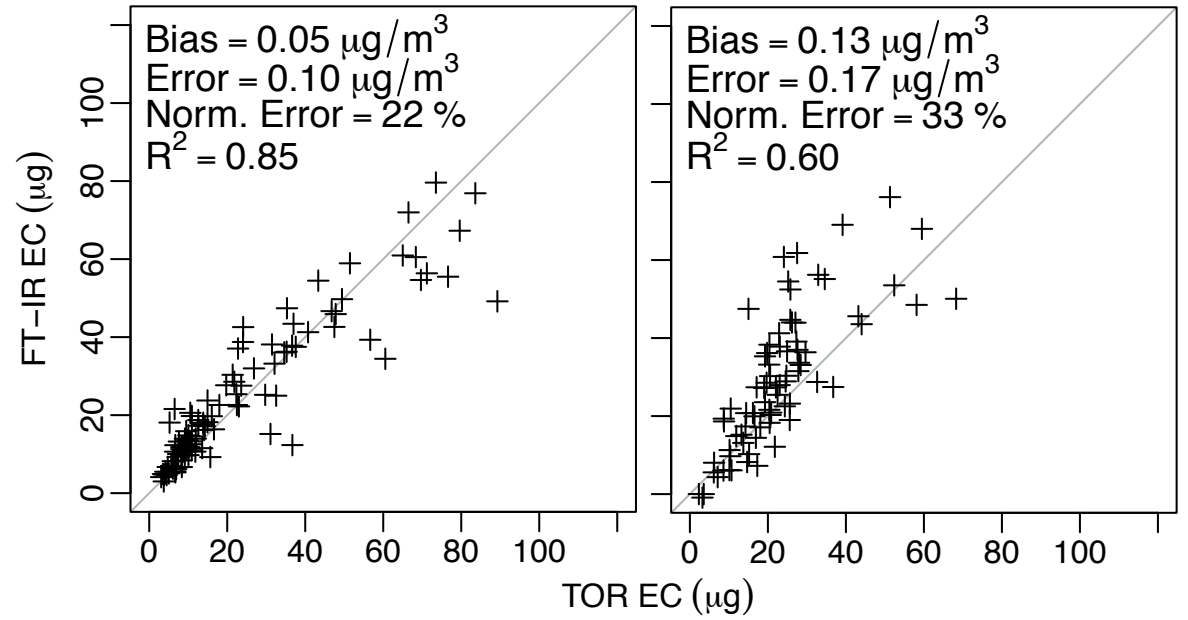

Figure S7. Scatterplot and performance metrics between FT-IR EC and TOR EC of the Korea and Fresno sites (Site ID 10 and 11 respectively) calibrated with the Calibration 2011 set. Concentration units of $\mu \mathrm{g} \mathrm{m}^{-3}$ for bias and error are based on the IMPROVE nominal volume of $32.8 \mu \mathrm{g} \mathrm{m}^{-3}$.

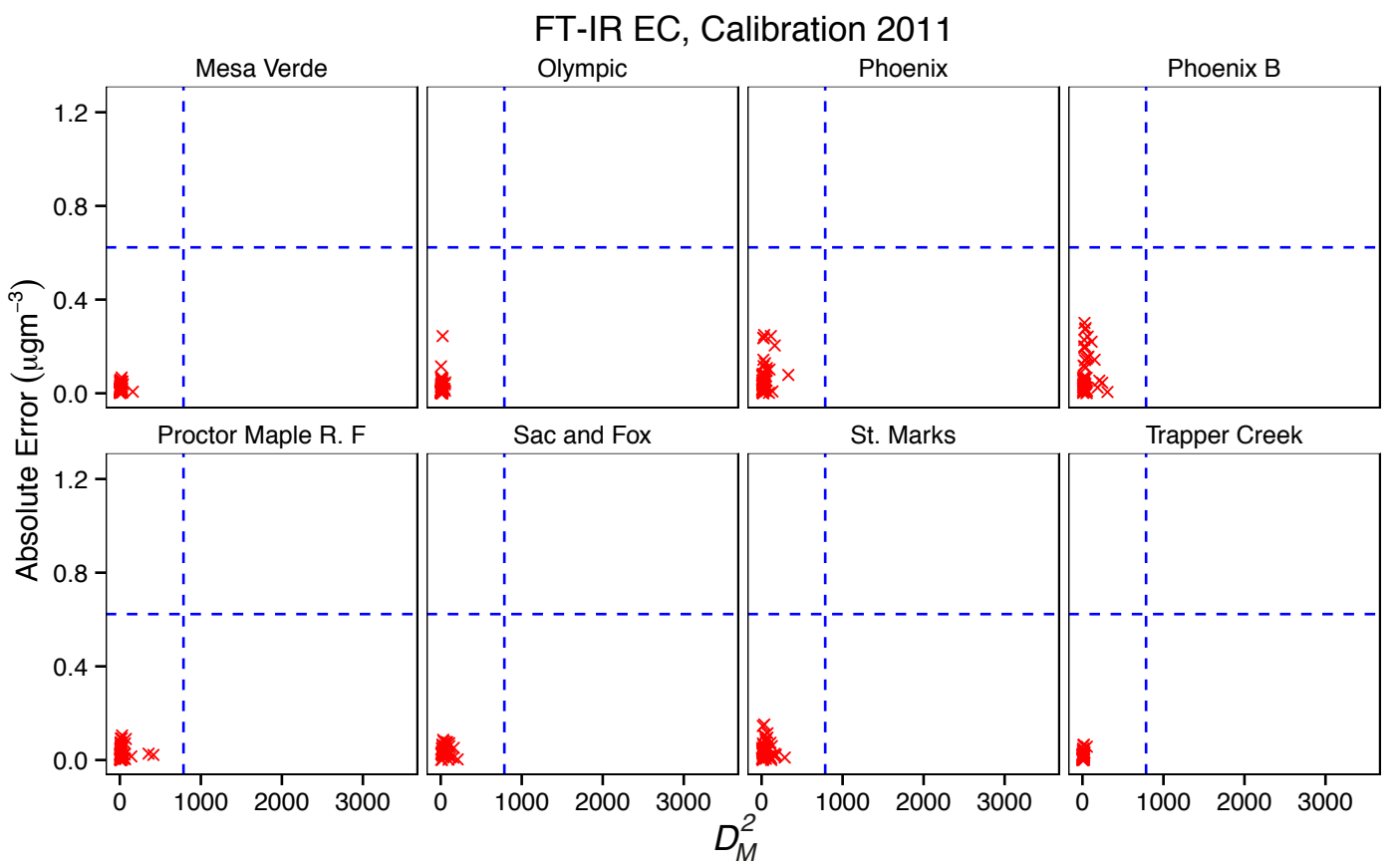

Figure S8. FT-IR OC, Calibration 2011. Anticipation of the prediction error. Mahalanobis distances per sample and site (between the scores of each dataset described in Section 2.1 and the centroid of the calibration dataset) against absolute errors (between TOR OC and FT-IR OC). Blue dashed lines represents the boundaries used for classification as explained in Section 2.4. 
FT-IR EC, Test 2011

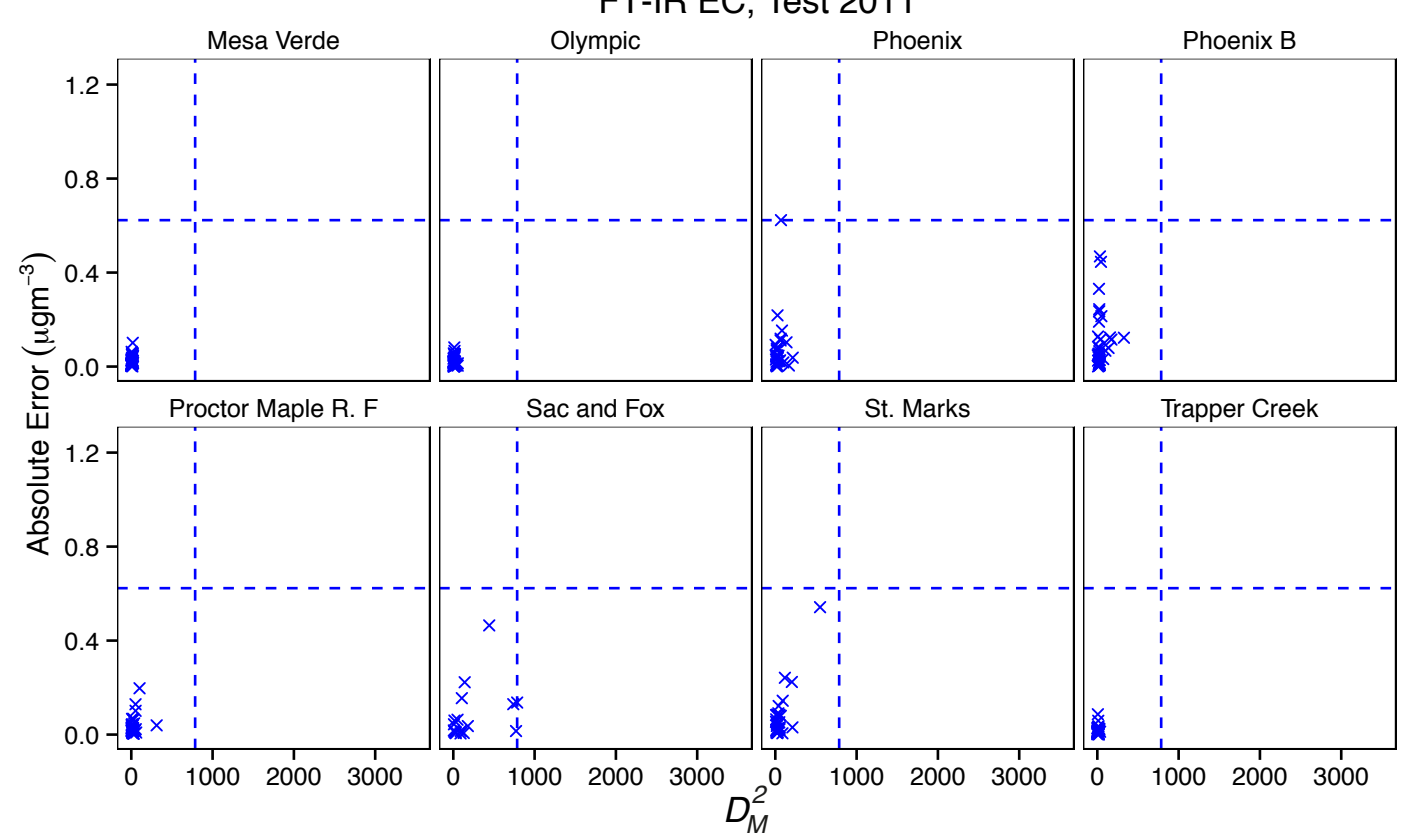

Figure S9. FT-IR OC, Test 2011. Anticipation of the prediction error. Mahalanobis distances per sample and site (between the scores of each dataset described in Section 2.1 and the centroid of the calibration dataset) against absolute errors (between TOR OC and FT-IR OC). Blue dashed lines represents the boundaries used for classification as explained in Section 2.4.

FT-IR EC, Test 2013

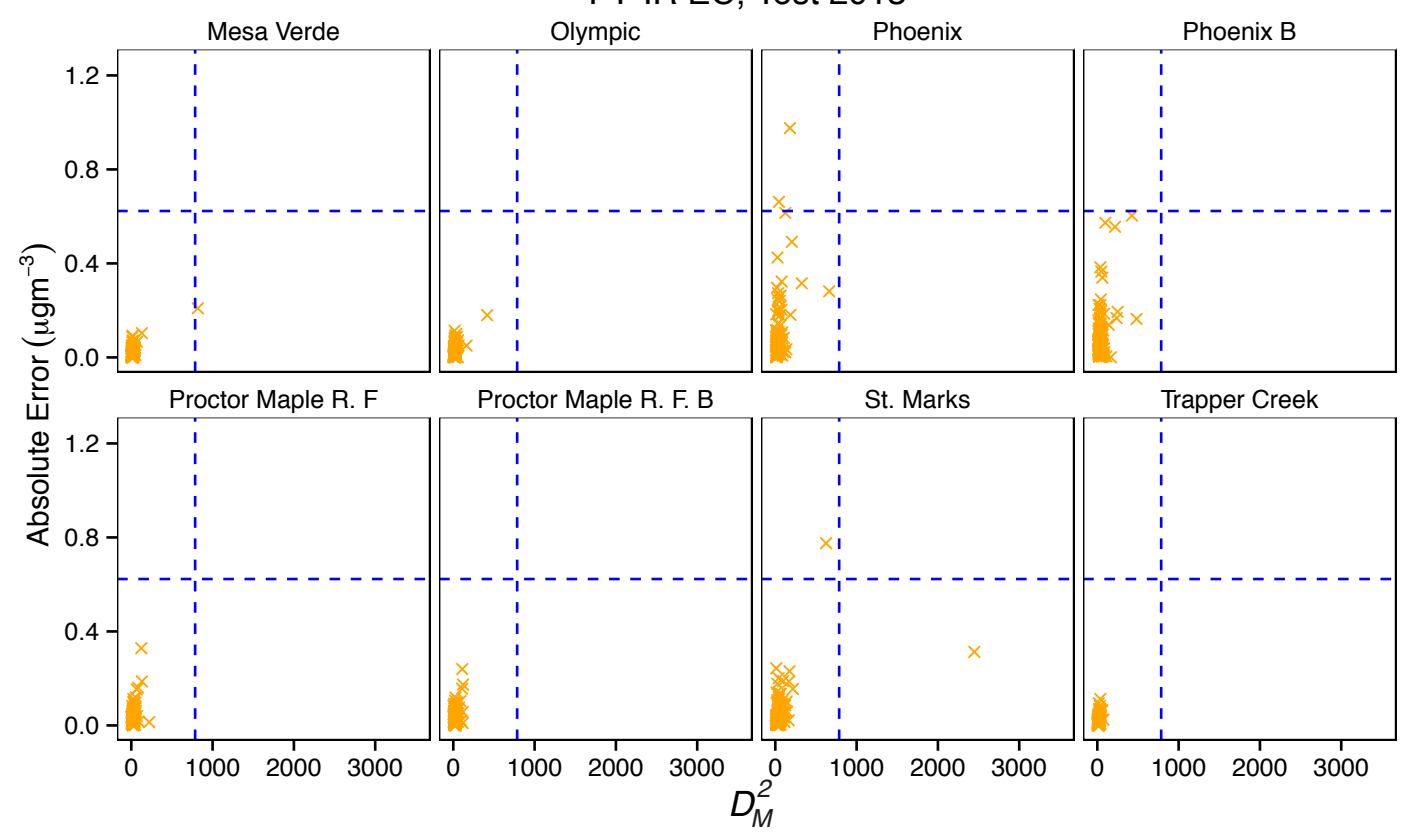

Figure S10. FT-IR OC, Test 2013. Anticipation of the prediction error. Mahalanobis distances per sample and site (between the scores of each dataset described in Section 2.1 and the centroid of the calibration dataset) against absolute errors (between TOR OC and FT-IR OC). Blue dashed lines represents the boundaries used for classification as explained in Section 2.4. 


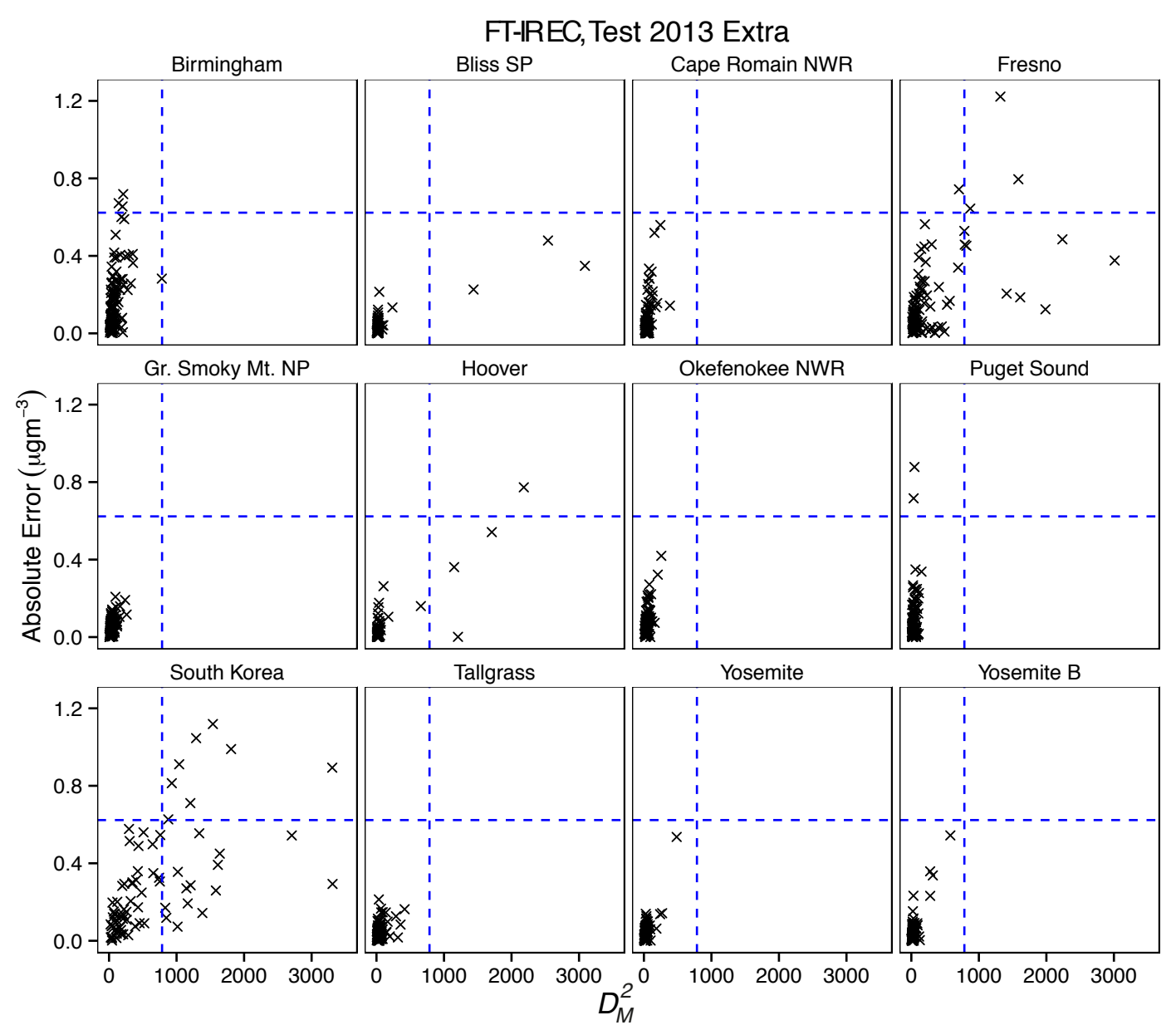

Figure S11. FT-IR OC, Test 2013 Addl. Anticipation of the prediction error. Mahalanobis distances per sample and site (between the scores of each dataset described in Section 2.1 and the centroid of the calibration dataset) against absolute errors (between TOR OC and FT-IR OC). Blue dashed lines represents the boundaries used for classification as explained in Section 2.4. 